\title{
ESCORPIONES VENENOSOS ENCONTRADOS EN CORRIENTES Y CHACO. CARACTERÍSTICAS DE LA INTOXICACIÓN.
}

Dra. Ana M. Torres ${ }^{1}$, Dr. Francisco J. Camargo ${ }^{1}$, Bioq. Nadia Filippis ${ }^{1}$, Esp. en Clínica Médica Verónica Mariana Soler ${ }^{2}$

Palabras claves: Tityus, escorpiones, venenos, accidente, toxinas.

\section{Resumen:}

Mediante el Proyecto de Voluntariado Universitario "Alerta roja: escorpiones!" aprobado por la SPU, hemos realizado charlas, recorridas y trabajos de laboratorio en Corrientes y Chaco, alertando sobre la toxicidad de los escorpiones, la forma de reconocer especies tóxicas, medidas de prevención y profilaxis. Asimismo se capturaron ejemplares para su identificación y estudio posterior. Hemos encontrado las especies de escorpiones venenosos Tityus trivittatus $y$ T. confluens en Corrientes y Chaco, extraído su veneno (promedio 0,26 y 0,21 $\mathrm{mg}$ por ejemplar respectivamente) y analizado la casuística de accidentes particularmente en Puerto Vilelas (Chaco) encontrando que el $100 \%$ refería dolor en el accidente, no requiriéndose en ninguno de los casos la derivación a centros de mayor complejidad.

\section{Abstract}

By University Volunteering Project "Red Alert: scorpions" approved by the SPU, we have conducted talks, traveled and laboratory work in Corrientes and Chaco, warning about the toxicity of scorpions, how to recognize toxic species, prevention and prophylaxis. Specimens for identification and further study is also captured. We found species of poisonous scorpions Tityus trivittatus y T. confluens in Corrientes and Chaco, extracted venom (average 0.26 and $0.21 \mathrm{mg}$ per specimen, respectively) and analyzed the casuistry of accidents, particularly in Puerto Vilelas (Chaco), we find that 100\% reported pain at the site of the bite, not requiring in either case referral to centers of greater complexity.

\section{Introducción}

En Argentina, el Orden Scorpiones está representado por más de 45 especies de escorpiones o alacranes pertenecientes a dos familias Buthidae y Bothriuridae, siendo los primeros los más peligrosos por la severidad de su picadura. Centraremos nuestra atención, particularmente en el género $T i-$ tyus, de mayor presencia en nuestra región. Mediante el Proyecto de Voluntariado Universitario "Alerta roja: escorpiones!" aprobado por la SPU, hemos realizado charlas, reco-

${ }^{1}$ Cátedra: Toxicología y Química Legal, Facultad de Ciencias Exactas y Naturales y Agrimensura. Universidad Nacional del Nordeste / 2 Centro de Salud Enrique Finochietto. Puerto Vilelas. Chaco

Dirección postal Dra. Torres, Ana María: Campus Deodoro Roca, Edificio de Química, $1^{\circ}$ piso ala este, laboratorio de Toxicología y Química Legal, FaCENA-UNNE.3400. Corrientes. amtorres@exa.unne.edu.ar. Cel.: 0379154753524 
rridas y trabajos de laboratorio en Corrientes y Chaco, alertando sobre la toxicidad de los escorpiones, la forma de reconocer especies tóxicas, medidas de prevención y profilaxis.

Según el Boletín Epidemiológico Periódico (BEP 2009), en los años 2001 al
Son escorpiones venenosos de color marrón claro o amarillento con tres líneas longitudinales oscuras en el lomo y raramente superan los $5 \mathrm{~cm}$ de longitud. Sus hábitos son principalmente nocturnos. Se mantienen activos durante todo el año, aunque con menor intensidad en las épocas más frías encontrándoselo en zonas rurales, domici-

Figura 1. Diferenciación entre escorpiones venenosos y no venenosos de la región

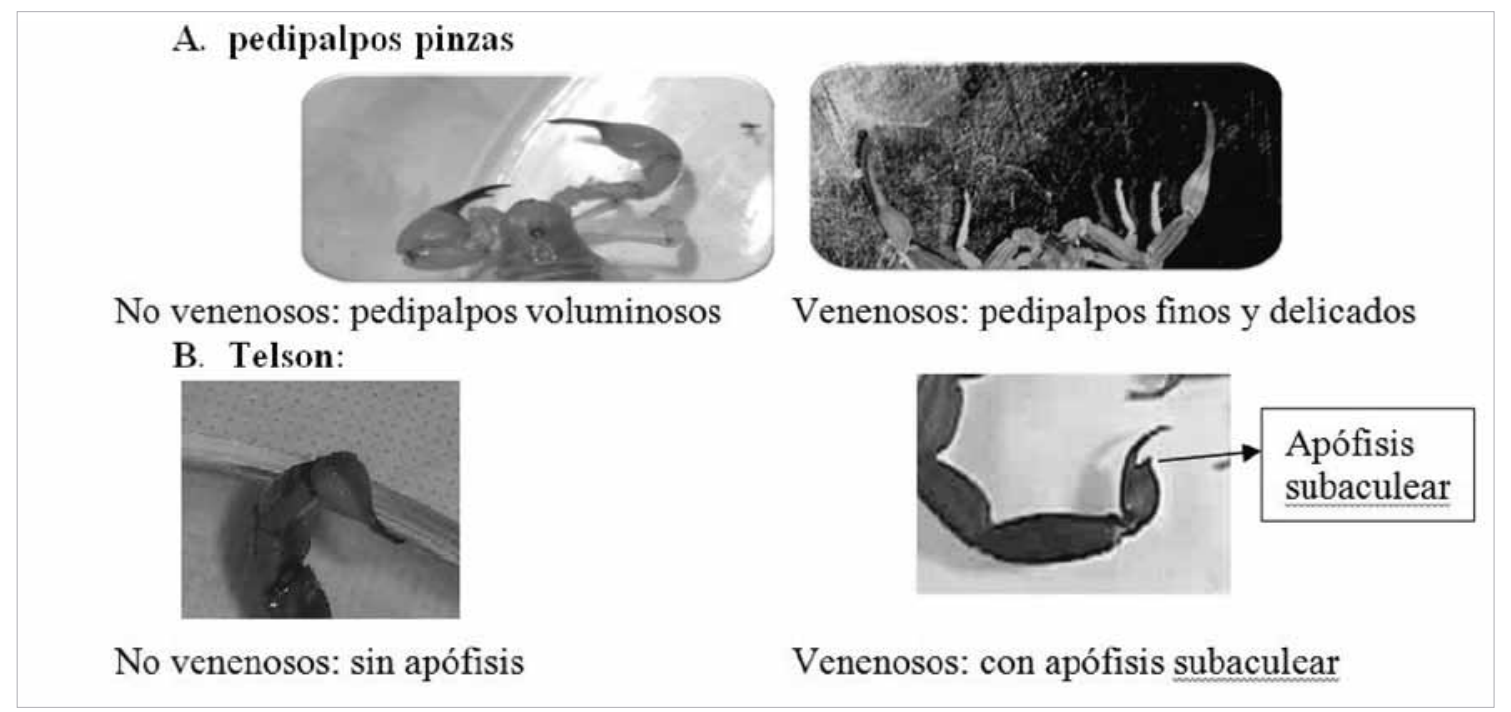

2008, se registraron 24 muertes (23 niños menores de 10 años), la mayoría de los casos reportados coinciden con los meses de febrero-marzo y noviembre-diciembre, con tendencia creciente en la región Noroeste, siendo la segunda más afectada la región Noreste de nuestro país. Es por lo tanto imprescindible reconocer las especies venenosas de las no venenosas, para lo cual se debe tener en cuenta las características del telson y pedipalpos como se indica en la figura 1 .

Entre las especies de importancia sanitaria de la región encontramos a Tityus trivittatus trivittatus (Figura 2-A); en $\mathrm{Co}^{-}$ rrientes, Entre Ríos, Chaco, Santa Fe, N. E. de Buenos Aires, E. de Santiago del Estero y E. de Córdoba. liarias y peridomiciliarias, con preferencia en lugares húmedos y oscuros donde abunden arañas e insectos, especialmente cucarachas (su alimento preferido) y grillos.

Otra especie tóxica es Tityus confluens (Figura 2-B), con dorso oscuro uniforme que lo diferencia de T. trivittatus. Iguales características generales y de toxicidad a $T$. trivittatus. Causante de envenenamientos severos y casos fatales.

Otra especie venenosa, Tityus babiensis (Figura 2-C) es típicamente brasileña, pero puede encontrarse en Argentina. De color negro, robusto, con patas y pedipalpos más claros. Tamaño: 60 - $70 \mathrm{~mm}$. Se los encuentra en campos, plantaciones y bosques. 
Figura 2. Especies de escorpiones venenosos: A. T. trivittatus; B. T. confluens; C. T. babiensis; D. T. serrulatus. Especie de escorpión no venenoso: E. T. elegans

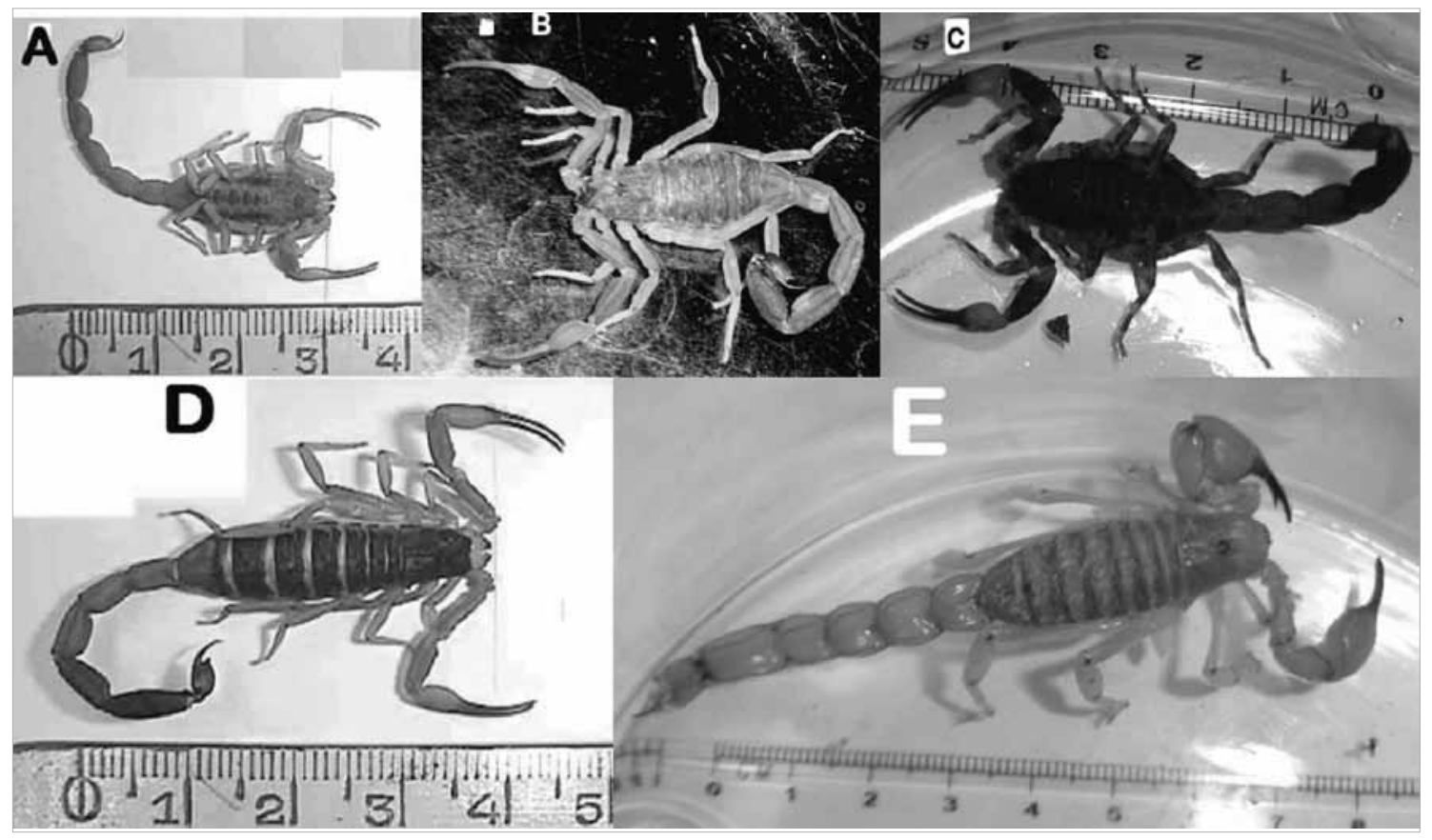

Con relación a Tityus serrulatus (Figura 2-D), Camargo et al 2000 alertan sobre la presencia de ejemplares de esta especie en la ciudad de Corrientes, de importancia médica en Brasil y posiblemente en toda América del Sur por su veneno neurotóxico muy activo. De color amarillo uniforme, dorso marrón oscuro, 60- $70 \mathrm{~mm}$ de longitud, contextura robusta y cola aserrada. Son escorpiones domiciliarios y peridomiciliarios. Se los encuentra dentro de las ciudades y construcciones humanas (Bücherl W. 1953).

Entre las especies sin importancia sanitaria de la región (no venenosas) podemos encontrar a Timogenes elegans (Figura 2-E), de color amarillento y líneas transversales más oscuras. Pedipalpos y contextura general robusta. Telson: sin apófisis subaculear. Tamaño: $100 \mathrm{~mm}$. Encontrado en
General Pinedo y Charata (Charata), de hábitat subterráneo.

\section{Resultados}

Se logró la captura e identificación de las siguientes especies:

T.trivittatus: barrios Camba Cuá, Centro, La Rosada, Madariaga, Dr. Nicolini, Islas Malvinas, Gral. San Martín y Progreso (Corrientes). Puerto Vilelas y Quitilipi (Chaco).

T. confluens: parte del barrio Camba Cuá, Deportes, parte del barrio La Rosada, San Gerónimo, Campus y Cacique Canindeyú (Corrientes). Puerto Vilelas y Quitilipi (Chaco)

T.bahiensis: se encontró en tronco de árboles en la localidad de Yapeyú, Ctes. 
Se extrajo veneno de las dos especies más abundantes, las cantidades promedio extraídas por ejemplar mediante pinza eléctrica, fueron de $0,26 \mathrm{mg}$ para $T$. trivittatus y $0,21 \mathrm{mg}$ para $T$. confluens. El perfil electroforético fue similar para ambas especies, siendo sus componentes mayoritarios de naturaleza polipeptídica, de $4 \mathrm{kda}, 27 \mathrm{kda}$ $\mathrm{y}>38 \mathrm{kda}$ (calculados teniendo en cuenta la movilidad de los patrones de PM), lo cual coincide con lo expuesto por Becerril et al., 1997, Verano Braga et al., 2008, entre otros.

El bajo peso molecular de los componentes de los venenos escorpiónicos justifica y explica el tropismo que poseen estas toxinas por el sistema nervioso

Es importante destacar que en el ser humano, el veneno actúa a distintos niveles, destacando particularmente su acción sobre el sistema respiratorio donde provoca un aumento en las secreciones bronquiales pudiendo llegar, en los casos más severos, a la obstrucción; como así también sobre el sistema cardiovascular donde la manifestación más comúnmente asociada al accidente es un marcado y sostenido cuadro hipertensivo.

Según datos estadísticos del Instituto Butantan (Magalhaes et al., 1998), cuando se produce un accidente escorpiónico, las regiones del cuerpo más frecuentemente picadas son: manos 57\%; brazos 7\%; pies 18\%; piernas 3,5\%; otros sitios 14,5\%. Existen relevamientos realizados por Amarilla et al., 2008 y por De Roodt et al., 2003 que coinciden con el dolor como el hallazgo más común en este tipo de accidentes.

Según nuestra experiencia, a partir de los datos obtenidos del relevamiento realizado para un total de 17 accidentes provocados por picadura de escorpión atendidos en el
Centro de Salud de Puerto Vilelas "Enrique Finochietto", Chaco; se encontró que el rango de edad de los individuos que habían sufrido el accidente se encontraba comprendido entre los 12 a 37 años. El $41 \%$ de los accidentes escorpiónicos ocurrió en el sexo femenino, de las cuales un $43 \%$ se encontraba cursando un embarazo, en diferentes semanas de gestación. E1 59 $\%$ fueron varones. $\mathrm{El}$ 6\% de los casos consultó recién 12 hs posteriores a la ocurrencia del accidente escorpiónico. E1 100\% de los pacientes refirieron dolor y ardor en el sitio de la picadura, presentado solo $12 \%$ de los casos hipertensión arterial. Los sitios de picadura, en orden de frecuencia fueron: miembros superiores (dedos de manos), miembros inferiores (dedos de pies), tórax (mama y axila) y cabeza (ojos). Ninguno de los casos tuvo que ser derivado para internación a un centro de mayor complejidad. Todos los pacientes recibieron corticoides por vía parenteral, paracetamol vía oral y difenhidramina; este último medicamento fue obviado en las pacientes que cursaban su primer trimestre de embarazo.

Entre las posibles formas de presentación del cuadro clínico del accidente escorpiónico, los autores coinciden en que existen dos formas básicas: un síndrome local, caracterizado por dolor, eritema localizado, edema, sensación de adormecimiento o anestesia alrededor del sitio de la picadura, contracciones musculares fibrilares; y un síndrome sistémico o generalizado; caracterizado por piel pálida y sudorosa, cefalea - vértigo - astenia, estado de agitación o somnolencia, sialorrea - rinorrea, taquicardia - taquipnea, hipertensión arterial o hipotensión arterial franca en el caso de shock.

El diagnóstico sólo es fácil si se puede identificar al animal agresor. Por ello resulta 
del mayor interés una tríada de signos con gran valor diagnóstico que, casi invariablemente, asociada al accidente escorpiónico, ella es: sialorrea-rinorrea-lagrimeo. Siempre que esta tríada se presente, y ante la ausencia de otra evidencia, deberá sospecharse la ocurrencia de un accidente escorpiónico. A esta tríada debe agregársele otro síntoma de fundamental importancia, el dolor. Este puede ser de intensidad variable y de tipo quemante.

El tratamiento general contempla una serie de medidas inespecíficas tendientes a aliviar las molestias que sufre el individuo afectado; ellas consisten en colocar compresas frías sobre el área afectada, uso de analgésicos (paracetamol), colocar vacuna antitetánica si no tiene esquema completo, administrar antihipertensivos de vida media / corta (NPS, NTG).

El tratamiento especifico consite en la administración de suero antiveneno escorpionico distribuído por el Instituto Nacional de Producción de Biológicos del ANLIS “Carlos Malbrán” a todas las provincias que notifican casos y lo soliciten, sobre todo en casos de pacientes menores de 15 años o mayores de 65 años, con antecedentes de accidentes previos con escorpiones, con grados de intoxicación III y IV. Siendo importante que se implemente dentro de los 15 minutos de ocurrido el accidente.

Las medidas de prevención consisten en mantener limpios y libres de escombros los baldíos próximos a la vivienda. Realizar fumigaciones periódicas con piretroides solos (deltametrina) o combinados con carbamatos. Cubrir los espacios debajo de las puertas y en ventanas para evitar que ingresen a los domicilios, control de las cámaras subterráneas, cañerías, sótanos y oquedades de las paredes en los que pueden encontrarse. Asimismo, y principalmente, deben controlarse las entradas y salidas de cañerías de las casas así como las aberturas y hendiduras por donde estos podrían ingresar a la casa o moverse entre los compartimentos. Para eso se deberá proceder al sellado de grietas, la colocación de rejillas adecuadas en los desagües y de tapas adecuadas en las salidas y entradas de cañerías.

Si ya ha detectado la presencia de escorpiones en la vivienda, evitar dejar prendas y calzados en el suelo durante la noche. Sacudir toda vestimenta antes de colocársela ya que los escorpiones pican cuando se sienten agredidos.

\section{Conclusión}

La realización del presente proyecto de voluntariado universitario ha resultado sumamente enriquecedor para los profesionales y alumnos que han participado del mismo, logrando obtener información de primera mano sobre esta problemática a partir del contacto directo con la población de diferentes localidades de nuestra región. Asimismo, se ha podido transmitir a la población afectada por esta problemática, nuestra experiencia en lo atinente a identificación, primeros auxilios, prevención y tratamiento de este tipo de accidentes. 


\section{Bibliografia}

Amarilla A., Jiménez M., Sosa Echeverría M., Servin R. Accidentes producidos por escorpiones en niños de la ciudad de Corrientes. Revista de posgrado de la VI Cátedra de Medicina. (2008) 177: 6-12

Becerril B., Marangoni S., Posan L.. Toxins and genes isolated from scorpions of the genus. Tityus. Toxicon (1997). 35 (6): 821-35.

Boletín Epidemiológico Anual. Ministerio de Salud. Dirección de Epidemiología, Ministerio de Salud de la Nación. Av. 9 de Julio 1925, Piso 9o (C1073ABA) Cdad. Autónoma de Buenos Aires, República Argentina. (2009) 22-25

Bücherl W. Escorpiões e escorpionismo no Brasil. Mem. Inst. Butantan (1953).25 (1): 53 - 82.

Camargo F., Ricciardi A. Sobre la presencia de un escorpión Tiyus serrulatus Lutz e Mello (Scorpiones, Buthidae) en la ciudad de Corrientes. Comunicaciones científicas y Tecnológicas. (2000). UNNE

De Roodt A. R. Recomendaciones para la Identificación, captura, mantenimiento y transporte de ejemplares de Tityus trivittatus. Area Investigación y Desarrollo / Serpentario Instituto Nacional de Producción de Biológicos - A.N.L.I.S. "Dr. Carlos G. Malbrán” Ministerio de Salud y Ambiente. Secretaría de Regulación, Relaciones y Políticas Sanitarias.

De Roodt A., García S., Salomón O., Segre L., Dolab J., Funes R., De Titto E. Epidemiological and clinical aspects of scorpionism by Tityus trivittatus in Argentina. Toxicon. (2003) 41 (8): 971-7.

Magalhães M., Viana G., Anates R., Santos T., Cunha- Melo R. The mouse as na experimental model for Tityus serrulatus scorpion envenoming. Acta Cir. Bras. (1998) 13 (4): 205-210.

Verano-Braga, Rocha-Resende, Silva D., Ianzer D., Martin-Eauclaire M., Bougis P., De Lima M., Santos R., Pimenta A. Tityus serrulatus hypotensins: a new family of peptides from scorpion venom. Biochem Biophys Res. Común. (2008) 4; 371 (3): 515-20.

\section{Agradecimientos:}

A la SPU que confió y apoyó al Proyecto de Voluntariado Universitario Alerta roja: escorpiones! Reso-

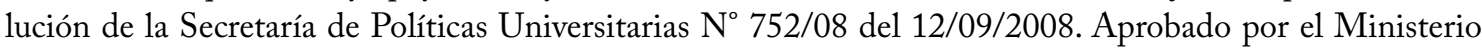
de Educación de la Nación.

Por el apoyo brindado: a la FaCENA, UNNE, Municipalidad de Puerto Vilelas (que declarara de Interés Municipal este Proyecto), Ministerio de Salud de la Provincia de Corrientes y al Hospital y Municipalidad de Quitilipi.

A los alumnos de la Carrera de Bioquímica: Álvarez Darío, Leiva Flavia Lorena, Arrieta Patricia del Carmen, Marturet Analía, Carrocino Jessica, Ramirez José Ignacio, Elias María Gabriela, Reigemborn Camila, Femenía Clementi María Victoria, Filippis Nadia, Salerno Hilda Paola, Sosa María de los Ángeles, Galfrascoli Soledad, Hidalgo Melisa, Langton Sofía. Por su entusiasmo, compromiso y acompañamiento en este Voluntariado.

En memoria al Prof. Armando I. A. Ricciardi y la Prof Ada E. Agrelo de Nassiff que nos acompañaron en la realizacion de este Proyecto. 